\title{
The campylobacteriosis conundrum - examining the incidence of infection with Campylobacter sp. in Australia, 1998-2013
}

\author{
C. R. M. MOFFATT ${ }^{1 *}, \mathrm{~K} \cdot \mathrm{GLASS}^{1}, \mathrm{R} \cdot \mathrm{STAFFORD}^{2}, \mathrm{C}^{\prime} \mathrm{D}^{\prime} \mathrm{ESTE}^{1}$ AND \\ M. D. $\mathrm{KIRK}^{1}$ \\ ${ }^{1}$ National Centre for Epidemiology and Population Health, Research School of Population Health, Australian \\ National University, Canberra, Australia \\ ${ }^{2}$ OzFoodNet, Communicable Diseases Branch, Queensland Health, Brisbane, Australia
}

Received 8 September 2016; Final revision 28 October 2016; Accepted 11 November 2016;

first published online 12 December 2016

\section{SUMMARY}

Campylobacter sp. are a globally significant cause of gastroenteritis. Although rates of infection in Australia are among the highest in the industrialized world, studies describing campylobacteriosis incidence in Australia are lacking. Using national disease notification data between 1998 and 2013 we examined Campylobacter infections by gender, age group, season and state and territory. Negative binomial regression was used to estimate incidence rate ratios (IRRs), including trends by age group over time, with post-estimation commands used to obtain adjusted incidence rates. The incidence rate for males was significantly higher than for females [IRR $1 \cdot 20,95 \%$ confidence interval (CI) $1 \cdot 18-1 \cdot 21$ ], while a distinct seasonality was demonstrated with higher rates in both spring (IRR 1·18, 95\% CI 1·16-1·20) and summer (IRR 1·17, 95\% CI 1.16-1·19). Examination of trends in age-specific incidence over time showed declines in incidence in those aged $<40$ years combined with contemporaneous increases in older age groups, notably those aged 70-79 years (IRR 1998-2013: 1·75, 95\% CI 1·63-1·88). While crude rates continue to be highest in children, our findings suggest the age structure for campylobacteriosis in Australia is changing, carrying significant public health implications for older Australians.

Key words: Campylobacter, epidemiology, foodborne infections, foodborne zoonoses, incidence.

\section{INTRODUCTION}

Campylobacter sp. are internationally significant pathogens, causing an estimated 96 million cases of foodborne illness annually [1]. Campylobacteriosis presents as an acute self-limiting enteritis, characterized by watery diarrhoea, abdominal pain and fever, with symptoms lasting for about 1 week [2]. In addition, Campylobacter infections can result in a number of

\footnotetext{
* Author for correspondence: Mr C. R. M. Moffatt, National Centre for Epidemiology and Population Health, Research School of Population Health, Australian National University, Canberra, Australia, 2602.

(Email: cameron.moffatt@anu.edu.au)
}

extra-intestinal manifestations that include bacteraemia, Guillain-Barré syndrome and reactive arthritis [3].

Despite campylobacteriosis being one of the most common causes of foodborne illness in industrialized countries, outbreaks of disease are less frequently reported, with most cases thought to be sporadic, in that they are otherwise unrelated to each other [4]. Poultry are recognized as a major reservoir for Campylobacter sp., leading to contamination along the production chain and transmission to humans through contaminated meat at the retail level [5]. Other domesticated animals including cattle and pigs, and environmental sources such as contaminated water, also play a role in the transmission of infection to humans [3]. 
In Australia, the crude incidence of Campylobacter infections was 115.8 cases $/ 100000$ population or 17 733 notifications in 2011 [6]. As such, Australia has one of the highest rates of infection in the developed world; being ten times that of the United States, double that seen in the European Union, but about 25\% less than in New Zealand [5]. International data also suggests changes in the age structure of campylobacteriosis, with increasing and decreasing rates of incidence reported across different age strata in England and the United States [7, 8].

Notifications, however, represent only a small proportion of all cases, with an estimate of underreporting in Australia showing that for every 100 notifications, 1001 (95\% credible interval 664-2251) cases of campylobacteriosis occur in the community [9]. Further Australian estimates have suggested that 234000 domestically acquired infections occur each year, including 3200 hospital admissions [10]. Despite the high incidence of campylobacteriosis, there have been no published studies describing the epidemiological patterns and national disease trends in Australia. To improve the understanding of the reported incidence of campylobacteriosis in Australia we compared the rates of infection by sex, age group, season, and Australian states and territories, including trends over time across age groups.

\section{METHODS}

In this study, we used national data on reported cases of campylobacteriosis in Australia to analyse disease incidence by gender, age group, season and state and territory from 1998 to 2013. Campylobacteriosis is a notifiable condition in all Australian states and territories, except New South Wales (NSW), with all states and territories, except NSW, having public health legislation mandating its reporting by doctors and/or pathology providers [11]. A confirmed case requires laboratory definitive evidence of the isolation or detection of Campylobacter sp. [12]. Since 1991, the states of Queensland, South Australia (SA), Tasmania, Victoria, and Western Australia (WA); and the two territories: the Australian Capital Territory (ACT) and the Northern Territory (NT) have all collected and reported core data on cases to the National Notifiable Diseases Surveillance System (NNDSS). Ethics approval for this study was granted by the Australian National University Human Research Ethics Committee.

\section{Data sources}

We requested de-identified campylobacteriosis NNDSS notifications (including 'notification receive date', 'true onset date', 'diagnosis date', 'age at onset', and 'sex') for each state and territory for 1991-2014 from the Communicable Disease Network of Australia (CDNA). Because of incompleteness of some NNDSS data fields we restricted our analysis to cases with diagnosis dates from 1998 to 2013 inclusive. We also removed 2014 data from our final analysis because of significant changes in diagnostic practices in Australian primary testing laboratories, namely the widespread introduction of culture-independent diagnostic methods. 'Diagnosis date' was used for all analyses, which is defined as illness onset date, or where onset date is unknown, the earliest of the specimen collection date, the notification date, or the date the notification is received. Detail on species and postcode locations for notifications was not requested.

Rates of illness/100 000 population were calculated using the mid-year (June quarter) estimated resident population by age and sex for each state and territory, for each year between 1998 and 2013 from the Australian Bureau of Statistics (ABS) [13, 14].

\section{Analysis}

The primary aim of our analysis was to examine the reported incidence of campylobacteriosis over time, across age groups and between states and territories. Age was categorized into the following groups for descriptive analysis < $<$ year, 1-4 years, 5-9 years and in 10-year age groups from 10-19 years until $\geqslant 80$ years. We defined seasons as summer (December, January, February), autumn (March, April, May), winter (June, July August) and spring (September, October, November). ABS population numbers, obtained as the mid-year (30 June estimate) by age, sex and state and territory for each year, were used as denominators in the calculation of population incidence rates. Initial descriptive analysis included calculation of crude incidence rates/100 000 population by age group, sex, and state. Mean age of males and females was compared by $t$ test. We then used negative binomial regression to estimate incidence rate ratios (IRR) with $95 \%$ confidence intervals (CIs) by sex, season, state, and trend over time by age group. $P$ values from the $Z$ statistic for the null hypothesis that the coefficient was zero are also 

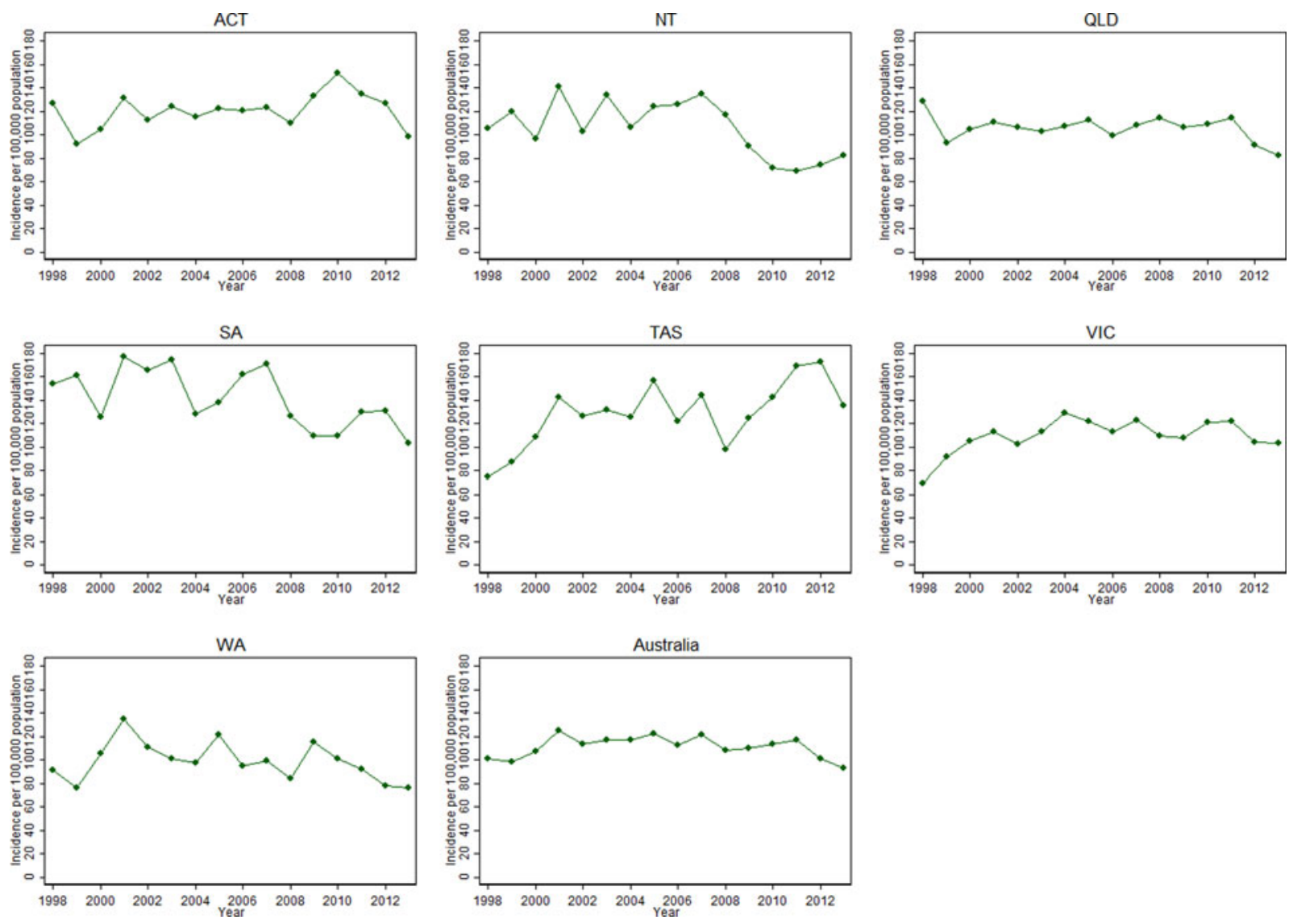

Fig. 1. Crude incidence rates for campylobacteriosis for Australia and individual states and territories, $1998-2013$.

presented. We assumed a linear time trend for incidence rates, using the interaction between age group and year to produce a trend over time, with year defined as the year of diagnosis and treated as a continuous variable. We also used post-estimation commands to estimate incidence rates for sex, state and year and total changes in incidence from 1998 to 2013 by age group. For the final model, 10-year age groups from $0-9$ years until $\geqslant 80$ years were used as a reduced number of age groups yielded a better model based on both Akaike's Information Criterion and Bayesian Information Criterion. All statistical analyses were performed using Stata v.14 (StataCorp, USA).

\section{RESULTS}

\section{Descriptive analysis}

There were 246151 notifications to NNDSS of campylobacteriosis with diagnosis dates between 1 January 1998 and 31 December 2013. Complete data was obtained on the state or territory of residence of all these cases. Age at onset data was missing for $451(0 \cdot 18 \%)$ cases, with data on gender missing for $605(0 \cdot 25 \%)$ cases. There were $72(0 \cdot 03 \%)$ cases where both age at onset and gender data were missing. In total, $1128(0 \cdot 46 \%)$ cases were excluded from the final dataset, giving a total of 245023 campylobacteriosis cases for inclusion in the analysis. The male: female ratio was $1 \cdot 2: 1$, with males comprising 133 $187(54 \cdot 4 \%)$ of cases. The mean age at onset for campylobacteriosis was 33.9 years (s.D. $=23 \cdot 28$, minimum $<1$, maximum 105 years). For females, the mean age was $35 \cdot 1$ years (S.D. $=23 \cdot 31$, minimum $<1$, maximum 105 years), and for males it was 32.9 years (s.D. $=$ $23 \cdot 20$, minimum $<1$, maximum 105 years) ( $t$ test $24 \cdot 3, P<0 \cdot 001)$.

Crude incidence rates for campylobacteriosis in Australia and for individual states and territories are shown in Figure 1. Nationally, crude incidence varied over time, with the highest annual rate seen in 2001 (124.7/100 000), decreasing to $92 \cdot 8 / 100000$ in 2013 . There was no obvious increasing or decreasing trend over time during that period. For states and territories crude incidence also varied over the period of 

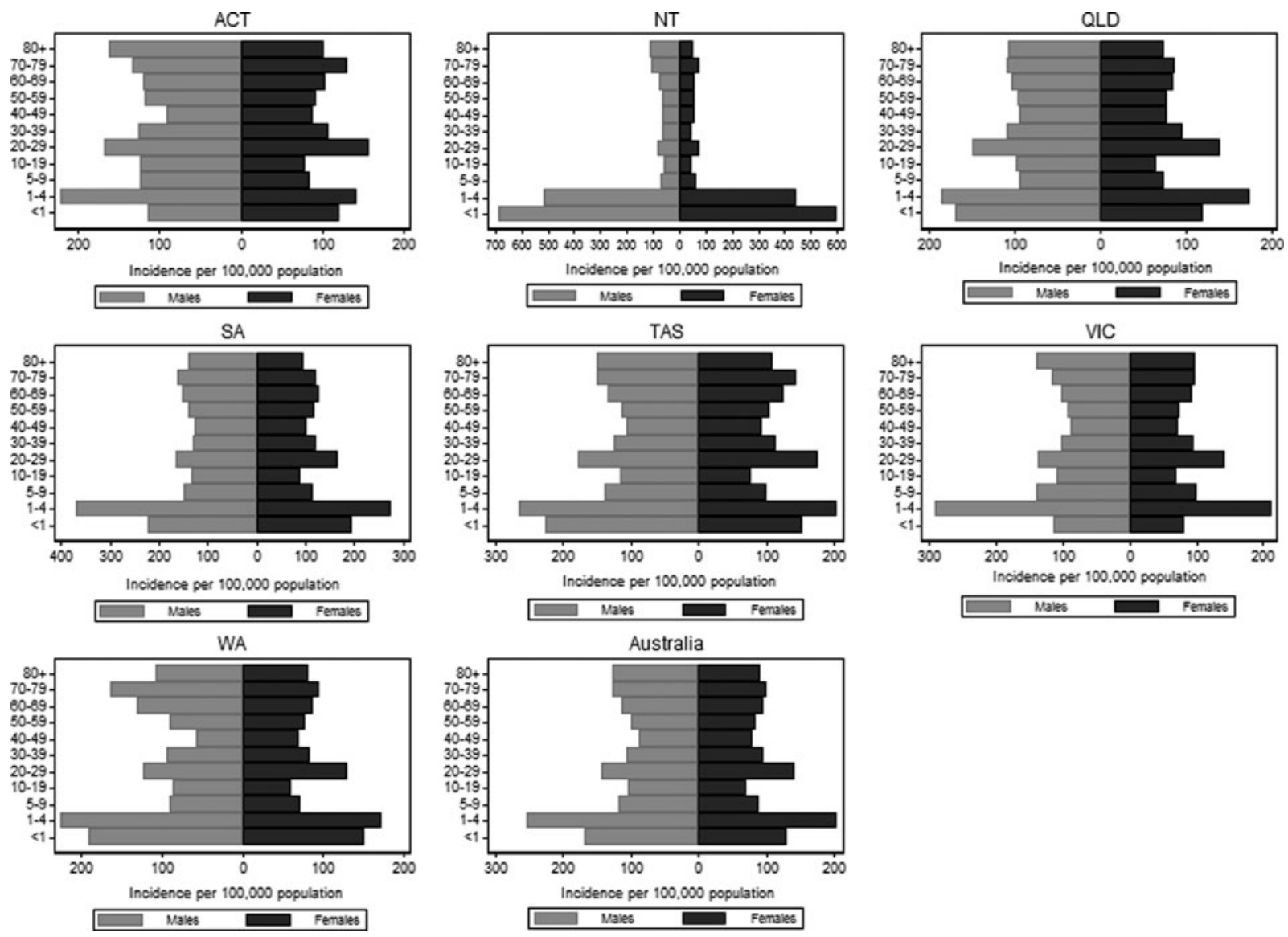

Fig. 2. Crude incidence rate pyramids by gender and age group for Australia and individual states and territories, 19982013.

reporting, being as high as $177 \cdot 5 / 100000$ in $\mathrm{SA}$ in 2001 and $172 \cdot 2 / 100000$ in Tasmania in 2012, to as low as 69.2/100000 in Victoria in 1998 and $66 \cdot 7 /$ 100000 in the NT in 2011.

Nationally the highest crude age-specific rates were observed in children aged between 1 and 4 years, with $254 \cdot 5$ cases/100000 population in males and $204 \cdot 2$ cases $/ 100000$ in females, with the lowest rates observed in females aged 10-19 years (69.7 cases/ 100000 population). Males had consistently higher rates of infection across all age groups. Crude sex and agespecific patterns of incidence were similar across most states and territories (Fig. 2). However, rates for NT children, in particular those aged $<5$ years, were markedly higher than elsewhere in Australia, reaching 684.7 cases/100 000 and 599.3 cases/100000 for males and females aged $<1$ year and 515.1/100000 and 445.7/ 100000 for males and females aged $1-4$ years, although these rates were determined on the basis of smaller case numbers in these sex and age strata than in other states and territories.

\section{Factors associated with incidence}

The rate of infection in males was $20 \%$ higher compared to females (Table 1: IRR $1 \cdot 20$, 95\% CI $1 \cdot 18$ 1.21). Nationally, notifications were shown to have a distinct seasonality, with similar rates in winter as autumn and statistically significant higher rates in both spring (IRR 1·18, 95\% CI 1·16-1·20) and summer (IRR $1 \cdot 17$, 95\% CI 1·16-1·19). Compared to the state of Victoria (the reference category), the highest IRR for Campylobacter infection was observed in the NT (IRR 1·40, 95\% CI 1·34-1·45), with significantly higher IRRs also observed in ACT, SA and Tasmania. Significantly lower IRRs for Campylobacter infection were observed in both Queensland (IRR 0.96, 95\% CI $0.95-0.98$ ) and WA (IRR 0.91, 95\% CI 0.89-0.93).

\section{Trends over time}

Examination of the interaction between age group and year showed significant differences in the trends over 
Table 1. Negative binomial regression for Campylobacter notifications by gender, season, state and age group and time, Australia 1998-2013

\begin{tabular}{|c|c|c|c|c|}
\hline & Notifications $(n=245023)$ & IRR & $95 \% \mathrm{CI}$ & $P$ value \\
\hline Gender & \multicolumn{4}{|c|}{ (Reference: female, $n=111836$ ) } \\
\hline Male & 133187 & $1 \cdot 20$ & $1 \cdot 18-1 \cdot 21$ & $<0 \cdot 001$ \\
\hline Season & \multicolumn{4}{|c|}{ (Reference: autumn, $n=55972$ ) } \\
\hline Winter & 56518 & $1 \cdot 01$ & $0 \cdot 99-1 \cdot 03$ & $0 \cdot 26$ \\
\hline Spring & 66135 & $1 \cdot 18$ & $1 \cdot 16-1 \cdot 20$ & $<0 \cdot 001$ \\
\hline Summer & 66398 & $1 \cdot 17$ & $1 \cdot 16-1 \cdot 19$ & $<0 \cdot 001$ \\
\hline State & \multicolumn{4}{|c|}{ (Reference: Victoria, $n=89369$ ) } \\
\hline Australian Capital Territory & 6581 & $1 \cdot 31$ & $1 \cdot 27-1 \cdot 35$ & $<0 \cdot 001$ \\
\hline Northern Territory & 3576 & $1 \cdot 40$ & $1 \cdot 34-1 \cdot 45$ & $<0 \cdot 001$ \\
\hline Queensland & 67193 & 0.963 & $0 \cdot 948-0 \cdot 978$ & $<0 \cdot 001$ \\
\hline South Australia & 35234 & $1 \cdot 31$ & $1 \cdot 29-1 \cdot 33$ & $<0 \cdot 001$ \\
\hline Tasmania & 10145 & $1 \cdot 31$ & $1 \cdot 28-1 \cdot 35$ & $<0 \cdot 001$ \\
\hline Western Australia & 32925 & $0 \cdot 910$ & $0 \cdot 894-0.926$ & $<0 \cdot 001$ \\
\hline \multicolumn{5}{|c|}{ Total change in incidence by age group, 1998-2013 } \\
\hline $0-9$ years & 46944 & $0 \cdot 63$ & $0 \cdot 60-0.66$ & $<0 \cdot 001$ \\
\hline $10-19$ & 26160 & $0 \cdot 97$ & $0 \cdot 92-1 \cdot 03$ & $0 \cdot 35$ \\
\hline $20-29$ & 45195 & $0 \cdot 78$ & $0 \cdot 74-0 \cdot 81$ & $<0 \cdot 001$ \\
\hline $30-39$ & 32972 & $0 \cdot 89$ & $0 \cdot 84-0.94$ & $<0 \cdot 001$ \\
\hline $40-49$ & 27639 & $1 \cdot 06$ & $1 \cdot 01-1 \cdot 12$ & $0 \cdot 03$ \\
\hline $50-59$ & 24954 & $1 \cdot 13$ & $1 \cdot 07-1 \cdot 20$ & $<0 \cdot 001$ \\
\hline $60-69$ & 19609 & $1 \cdot 36$ & $1 \cdot 28-1 \cdot 45$ & $<0.001$ \\
\hline $70-79$ & 13896 & $1 \cdot 75$ & $1 \cdot 63-1 \cdot 88$ & $<0.001$ \\
\hline$\geqslant 80$ & 7654 & $1 \cdot 72$ & $1 \cdot 57-1 \cdot 89$ & $<0.001$ \\
\hline
\end{tabular}

IRR, Incidence rate ratio; CI, confidence interval.

time in different age groups (Table 1, Supplementary Fig. S1). Adjusted rates of incidence between 1998 and 2013 decreased for younger age groups (i.e. those aged $<40$ years), while for older age groups there were increases in incidence of campylobacteriosis (Fig. 3). Significant declines over the 15-year study period were observed in children aged 0-9 years (IRR $0 \cdot 63,95 \%$ CI $0 \cdot 60-0 \cdot 66$ ). The largest increases over time were observed in older age groups, notably the 70-79 years (IRR $1 \cdot 75,95 \%$ CI $1 \cdot 63-1 \cdot 88$ ) and $\geqslant 80$ years (IRR $1 \cdot 72,95 \%$ CI $1 \cdot 57-1 \cdot 89$ ) age groups.

\section{DISCUSSION}

Considerable variations in the crude incidence of campylobacteriosis among Australian states and territories and between age groups over time were observed. While crude rates of disease remain highest in the very young, our findings support a shift in the age structure of campylobacteriosis in Australia, with IRRs increasing for older age groups over time. Further examination of predicted age-specific rates indicates a declining incidence in cases aged $<40$ years but combined with a concomitant rise in incidence in older age groupings, notably those aged 70-79 years. Our analysis also affirms male gender to be significantly associated with a higher incidence of infection, along with the occurrence of a distinct seasonality with incidence rising markedly over the Australian spring and summer.

It has been suggested that over the past decade there has been an increase in campylobacteriosis in higher-income countries [3]; however, examination of published incidence rates shows there are considerable inter-country variations in incidence as well as between regions within individual countries [15]. Increases in overall incidence have been observed in the European Union [16], the United States [17] and the UK [7] but Japan has reported no discernible change [18], while in New Zealand, long recognized as having highest rates of campylobacteriosis, marked declines in incidence have been observed following interventions in poultry primary production and processing [19]. In Australia, national incidence has remained high but with the direction of any national trend being more difficult to discern, in part because of the 'regional' variations between states and territories. Due to the size of the Australian continent there is 


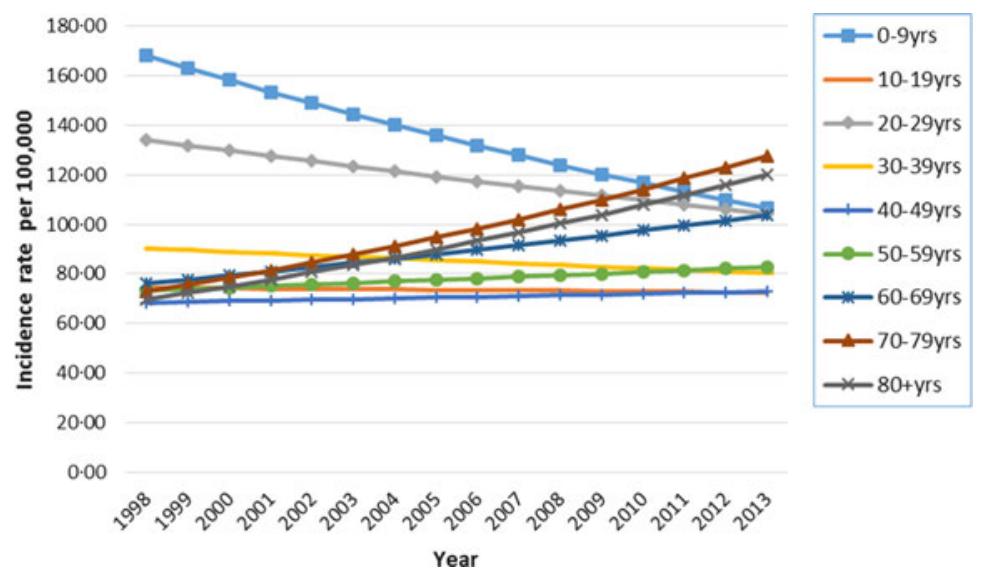

Fig. 3. Negative binomial regression margin plot for predicted incidence of campylobacteriosis by age group in Australia.

considerable climatic diversity. This ranges from tropical and monsoonal zones in the north, subtropical and temperate zones along the more populous eastern seaboard, smaller Mediterranean zones in the south and southwest, and a dominant semi-arid and desert zone covering the sparsely populated interior [20]. Given the seasonal patterns and climatic sensitivities of Campylobacter sp. [21] our findings of variations in incidence across regions of Australia are not wholly unexpected. Other explanations for the increases and decreases in both unadjusted and adjusted incidence over time might include differences in population exposures and behaviours, differences in laboratory detection and surveillance methods, differences in primary production practices and their regulation, or differences in relative contributions of assorted Campylobacter reservoirs to human disease [3].

Our findings from analysis of crude rates are broadly consistent with a number of studies from other high income countries that report a bimodal age-related pattern of incidence, with the highest infection rates in children aged $<5$ years, and with a second peak seen in younger adults aged between 20 and 29 years [22, 23]. These findings may be a reflection of differences in the relative contribution of environmental factors involved in the causal pathway of campylobacteriosis across these different age groups [24]. For example the high incidence shown for young children could reflect their more active exploration of their environment using hand-to-mouth behaviour [25], with the high incidence seen in NT children perhaps indicative of climatic differences or some other undefined environmental risks. In other age groups, poor hygiene practices as well as increased risky eating behaviour subsequent to moving out of the family home have been tendered as explainers for the peaks seen in young adults [26, 27]. A further consideration is the impact of acquired immunity on the epidemiology of campylobacteriosis [27].

While acknowledging a 'general' bi-modal pattern of age-related incidence for campylobacteriosis, we would also contend that in the Australian context our crude and adjusted incidence patterns actually demonstrate a tri-modal pattern, with a third incidence peak seen in those aged $>60$ years. Adults aged $>65$ years are recognized as being more susceptible than younger adults to certain enteric infections, including campylobacteriosis. A number of age-related factors may also contribute to their increased susceptibility and disease severity, including weakening of the immune system, changes to the gastrointestinal tract, a higher prevalence of comorbid conditions and more frequent use of antacids and immunosuppressant medication [28].

Our finding of a predicted increase in incidence over time among older adults is supported by analyses of data from England and Wales suggesting the age structure of campylobacteriosis has shifted, with older persons emerging as the population most at risk [7, 29]. In addition to the host factors previously described, social factors such as population ageing and demographic changes, including post-war population increases and the 'baby boom' of the 1960s may also play a role [29]. Given that the proportion of the Australian population aged $>65$ years is projected to increase from $14 \%$ ( $3 \cdot 2$ million) in 2012 to around $19 \%$ (5.8 million) in 2031 [30] any such change in the age structure of disease could carry significant health system implications. Studies of older persons infected with Campylobacter sp. show increased 
hospitalization and mortality, with clinical care further complicated by higher levels of comorbidities and risk for invasive infection [8, 31]. However, whether such a shift in the age structure of campylobacteriosis across high-income countries can be generalized is less certain, with a recent US study showing a decreasing rate of campylobacteriosis with increasing age [8].

That male gender was significantly associated with increased risk for infection in our study is a finding consistent with the epidemiology of Campylobacter in high-income country settings. This was further reflected in males having consistently higher rates of infection across all age groups, with the exception of those aged between 20 and 29 years [23, 32]. Explanations for the predilection towards males are not easily reconciled. Given the large differences in incidence between males and females in the youngest age groups where behavioural differences might be less pronounced, physiological differences may play a role in higher male disease incidence. This hypothesis is supported by an animal model showing both infection and shedding rates for $C$. jejuni to be greater in male mice [33].

Campylobacteriosis has been shown to exhibit a distinct seasonality in temperate regions, although clear reasons for this feature have proven difficult to ascertain [25]. Our finding, demonstrating a sharp rise in IRR in spring, with continuing and significantly elevated rates in summer is consistent with previous comparisons of high income countries in both the Northern and the Southern Hemispheres [21]. The consistency of a bacterial infection to exhibit summer peaks suggests the direct effect of a large scale environmental influence on a shared exposure route [34]. Although we did not examine any interaction between temperature and incidence, it is nevertheless plausible that increasing spring temperatures and the maintenance of high temperatures over summer greatly increase the risk for foodborne transmission of Campylobacter [35].

Poultry meat has been demonstrated to be the predominant foodborne source of campylobacteriosis in developed countries [3,5], with such findings arguably applicable to the Australian context, despite a current paucity of local source attribution data. In Australia, the poultry meat sector is particularly large and is dominated by two main producers, with primary processing occurring in all states [36]. In 2012, Australia introduced a national Primary Production and Processing Standard for poultry meat [37] but there is little publicly available data on what, if any, industry-initiated actions occurred between 1998 and 2013. Access to such information could have been used to model the effects of interventions on incidence. Given the increasing recognition that a 'One Health' approach, including greater cooperation and engagement by stakeholders is necessary to reduce the national burden of campylobacteriosis [38] we draw particular attention to the importance of data sharing between animal industries, agriculture and human health agencies to gain better understanding of campylobacteriosis in Australia. Australian stakeholders (e.g. veterinarians, scientists, policy makers, public health authorities, and industry) may benefit from undertaking closer examination of international successes, particularly in New Zealand, where integrated surveillance strategies have improved the understanding of disease drivers and contributed to a significant decline in disease incidence [39].

\section{Limitations}

There are a number of limitations to this study. Because campylobacteriosis is recognized as a largely sporadic infection, minimal public health data is collected for most cases and we were unable to exclude overseas acquired and outbreak associated cases from our analysis. Despite overseas travel previously being identified as a risk factor for infection in Australia, absolute numbers of cases with this risk have previously been reported as being small [40], leading us to conclude that most infections are likely to be domestically acquired. Similarly the impact of outbreaks on campylobacteriosis numbers is likely to be negligible given recognition that outbreaks are infrequent events relative to overall incidence [4]. Another potential limitation was an absence of comprehensive speciation data. We assumed that most infections were caused by $C$. jejuni and to a lesser extent $C$. coli. There are however indications that $C$. coli might contribute a larger number of infections than previously thought [41], with the importance of other emerging pathogenic species largely unknown in Australia. Similarly our dataset lacked geographic precision beyond the level of state or territory. Australia is a large and geo-climatically diverse continent and significant differences between urban and rural incidence have been demonstrated elsewhere internationally [15]. Such detail would be useful in further exploring differences in age, gender and origin of infection. Finally the increasing use by primary testing 
laboratories of culture-independent testing, notably nucleic acid tests, to diagnose enteric infections carries implications for disease surveillance and reporting with incidence rates likely to increase due to improved sensitivity in detection [42]. This latter point directly influenced our decision to exclude 2014 notifications from the analysis because of the widespread uptake of these methods across Australia in that year.

\section{CONCLUSIONS}

Our examination of the incidence of campylobacteriosis in Australia provides further illustration of the epidemiological complexities surrounding this common enteric pathogen. Australia continues to have a high rate of infection but considerable variation in incidence exists between sexes, age groups, and states and territories. While common or similar sources of transmission might be responsible for most disease across the different age groups, a transition to higher rates of disease in older persons carries significant implications for public health and clinical care. As such there is a need for improved understanding of risk factors specific to older Australians, combined with targeted public health messaging to educate this group of increased disease risk.

\section{SUPPLEMENTARY MATERIAL}

For supplementary material accompanying this paper visit https://doi.org/10.1017/S0950268816002909.

\section{ACKNOWLEDGEMENTS}

The data were collected as part of the Australian Research Council Linkage Project (LP110200431) 'Identifying and controlling the source of Campylobacter and Salmonella in Australia'. Cameron Moffatt receives funding from a National Health and Medical Research Council Public Health and Health Services Postgraduate Research Scholarship (APP1074790).

\section{DECLARATION OF INTEREST}

None.

\section{REFERENCES}

1. Kirk MD, et al. World Health Organization estimates of the global and regional disease burden of 22 foodborne bacterial, protozoal, and viral diseases, 2010: a data synthesis. PLoS Medicine 2015; 12: e1001921.
2. Allos BM. Campylobacter jejuni infections: update on emerging issues and trends. Clinical Infectious Diseases 2001; 32: 1201-1206.

3. Kaakoush NO, et al. Global epidemiology of campylobacter infection. Clinical Microbiology Reviews 2015; 28: $687-720$.

4. Unicomb LE, et al. Outbreaks of campylobacteriosis in Australia, 2001 to 2006. Foodborne Pathogens and Disease 2009; 6: 1241-1250.

5. Skarp CP, Hanninen ML, Rautelin HI. Campylobacteriosis: the role of poultry meat. Clinical Microbiology and Infection 2016; 22: 103-109.

6. OzFoodNet Working Group. Monitoring the incidence and causes of diseases potentially transmitted by food in Australia: Annual Report of the OzFoodNet network, 2011. Communicable Diseases Intelligence Quarterly Report 2015; 39: E236-264.

7. Gillespie IA, O'Brien SJ, Bolton FJ. Age patterns of persons with campylobacteriosis, England and Wales, 1990-2007. Emerging Infectious Diseases 2009; 15: 2046-2048.

8. Scallan E, et al. Bacterial enteric infections among older adults in the United States: Foodborne Diseases Active Surveillance Network, 1996-2012. Foodborne Pathogens and Disease 2015; 12: 492-499.

9. Hall G, et al. Estimating community incidence of Salmonella, Campylobacter, and Shiga toxin-producing Escherichia coli infections, Australia. Emerging Infectious Diseases 2008; 14: 1601-1609.

10. Kirk M, et al. Foodborne illness, Australia, circa 2000 and circa 2010. Emerging Infectious Diseases 2014; 20: 1857-1864.

11. Australian Government Department of Health. Links to state and territory public health legislation, the Quarantine Act, and the National Health Security Act 2007. Australian Government Department of Health, 2009.

12. Australian Government Department of Health. Campylobacteriosis case definition. Australian Government Department of Health, 2004.

13. Australian Bureau of Statistics. Australian Historical Population Statistics, 2014 (http://www.abs.gov.au/ausstats/abs@.nsf/mf/3105.0.65.001). Accessed 18 August 2015.

14. Australian Bureau of Statistics. Australian Demographic Statistics, December 2013 (http://www.abs.gov.au/ AUSSTATS/abs@.nsf/DetailsPage/3101.0Dec\%202013? OpenDocument). Accessed 18 August 2015.

15. Spencer SE, et al. The spatial and temporal determinants of campylobacteriosis notifications in New Zealand, 2001-2007. Epidemiology and Infection 2012; 140: 1663-1677.

16. European Food Safety Authority (EFSA), European Centre For Disease Prevention and Control (ECDC). The European Union Summary Report on Trends and Sources of Zoonoses, Zoonotic Agents and Food-borne Outbreaks in 2012. European Food Safety Authority Journal 2014; 12: 3547.

17. Crim SM, et al. Preliminary incidence and trends of infection with pathogens transmitted commonly through food-Foodborne Diseases Active Surveillance 
Network, 10 U.S. sites, 2006-2014. Morbidity and Mortality Weekly Report 2015; 64: 495-499.

18. Kumagai Y, et al. Estimating the burden of foodborne diseases in Japan. Bulletin of the World Health Organization 2015; 93: 540.

19. Sears A, et al. Marked campylobacteriosis decline after interventions aimed at poultry, New Zealand. Emerging Infectious Diseases 2011; 17: 1007-1015.

20. Stern H, De Hoedt G, Ernst J. Objective classification of Australian climates. Australian Meteorological Magazine 2000; 49: 87-96.

21. Kovats RS, et al. Climate variability and campylobacter infection: an international study. International Journal of Biometeorology 2005; 49: 207-214.

22. Nelson W, Harris B. Campylobacteriosis rates show age-related static bimodal and seasonality trends. New Zealand Medical Journal 2011; 124: 33-39.

23. Schielke A, Rosner BM, Stark K. Epidemiology of campylobacteriosis in Germany - insights from 10 years of surveillance. BMC Infectious Diseases 2014; 14: 30.

24. Arsenault $\mathbf{J}$, et al. Environmental characteristics associated with campylobacteriosis: accounting for the effect of age and season. Epidemiology and Infection 2012; 140: 311-322.

25. Strachan NJ, et al. Identifying the seasonal origins of human campylobacteriosis. Epidemiology and Infection 2013; 141: 1267-1275.

26. Byrd-Bredbenner C, et al. Risky eating behaviors of young adults-implications for food safety education. Journal of the American Dietetic Association 2008; 108: $549-552$.

27. Havelaar AH, et al. Immunity to Campylobacter: its role in risk assessment and epidemiology. Critical Reviews in Microbiology 2009; 35: 1-22.

28. Lund BM, O'Brien SJ. The occurrence and prevention of foodborne disease in vulnerable people. Foodborne Pathogens and Disease 2011; 8: 961-973.

29. Nichols GL, et al. Campylobacter epidemiology: a descriptive study reviewing 1 million cases in England and Wales between 1989 and 2011. BMJ Open 2012; 2.

30. Australian Bureau of Statistics. 3222.0-Population Projections, Australia, 2012 (base) to 2101 (http://
www.abs.gov.au/ausstats/abs@.nsf/Lookup/3222.0main +features $52012 \% 20$ (base) $\% 20$ to $\% 202101$ ). Accessed 18 August 2016.

31. Gradel KO, et al. Morbidity and mortality of elderly patients with zoonotic Salmonella and Campylobacter: a population-based study. Journal of Infection 2008; 57: 214-222.

32. Gillespie IA, et al. Demographic determinants for Campylobacter infection in England and Wales: implications for future epidemiological studies. Epidemiology and Infection 2008; 136: 1717-1725.

33. Strachan NJ, et al. Sexual dimorphism in campylobacteriosis. Epidemiology and Infection 2008; 136: 1492-1495.

34. Lal A, et al. Seasonality in human zoonotic enteric diseases: a systematic review. PLOS ONE 2012; 7: e31883.

35. D'Souza RM, et al. Does ambient temperature affect foodborne disease? Epidemiology 2004; 15: 86-92.

36. New South Wales Department of Primary Industries. NSW poultry meat industry overview, September 2015.

37. Food Standards Australia and New Zealand. Primary Production and Processing Standard for Poultry Meat (Standard 4·2 2). (FSANZ) FSAaNZ, ed. 422: FSANZ, 2012.

38. Golz G, et al. Relevance of Campylobacter to public health - the need for a One Health approach. International Journal of Medical Microbiology 2014; 304: 817 823.

39. Muellner P, et al. Molecular-based surveillance of campylobacteriosis in New Zealand - from source attribution to genomic epidemiology. Eurosurveillance 2013; 18.

40. Stafford RJ, et al. A multi-centre prospective casecontrol study of Campylobacter infection in persons aged 5 years and older in Australia. Epidemiology and Infection 2007; 135: 978-988.

41. Roux F, et al. Elucidating the aetiology of human Campylobacter coli infections. PLoS ONE 2013; 8: e64504.

42. Cronquist AB, et al. Impacts of culture-independent diagnostic practices on public health surveillance for bacterial enteric pathogens. Clinical Infectious Diseases 2012; 54 (Suppl. 5): S432-439. 